\title{
MODULI SPACES OF EINSTEIN METRICS ON 4-MANIFOLDS
}

\author{
MICHAEL T. ANDERSON
}

In this note, we announce some results showing unexpected similarities between the moduli spaces of constant curvature metrics on 2-manifolds (the Riemann moduli space) and moduli spaces of Einstein metrics on 4manifolds. Let $\mathscr{E}$ denote the moduli space of Einstein metrics of volume 1 on a compact, orientable 4-manifold $M^{4}$. If $\mathscr{M}_{1}$ denotes the space of smooth Riemannian metrics of volume 1 on $M$, endowed with a suitable smooth topology, then the diffeomorphism group $\mathscr{D}$ acts smoothly on $\mathscr{M}_{1}$ and $\mathscr{E}$ is the subspace of $\mathscr{M}_{1} / \mathscr{D}$ consisting of (equivalence classes) of Riemannian metrics $g$ satisfying the Einstein condition $E(g)=\operatorname{Ric}(g)-$ $(\lambda / 4) g=0$; here Ric denotes the Ricci curvature and $\lambda$ the scalar curvature. It is well known that $\lambda$ is a constant for Einstein metrics in dimension $\geq 3$. Concerning the coarse structure of $\mathscr{E}$, it is known [6] that $\mathscr{E}$ consists of countably many components $\mathscr{C}$, each of which is locally the quotient of a finite-dimensional real-analytic Hausdorff variety by a compact group action. The scalar curvature variable $\lambda: \mathscr{C} \rightarrow \mathbf{R}$ is constant on $\mathscr{C}$.

There is a natural Riemannian metric on $\mathscr{M}_{1}$, the $L^{2}$ metric, defined as follows: for $\alpha, \beta$ symmetric 2-tensors in $T_{g} \mathscr{M}_{1} \cong S^{2}(M)$, let $\langle\alpha, \beta\rangle=$ $\int_{M}(\alpha(x), \beta(x))_{g} d v_{g}(x)$, where $(,)_{g}$ is the inner product on $S^{2}(M)$ induced by $g$ and $d v_{g}$ is the volume form given by $g$. This induces a Riemannian metric on $\mathscr{M}_{1} / \mathscr{D}$ and thus a metric on the components $\mathscr{C}$ (since $\mathscr{C}$ is real-analytically path connected). Note however that the $L^{2}$ metric on $\mathscr{M}_{1} / \mathscr{D}$ is never locally complete (i.e. small metric balls are not complete).

In dimension 2, Einstein metrics are naturally considered to be metrics of constant scalar curvature. Thus, $\mathscr{E}$ is exactly the space of constant curvature metrics, or equivalently, the space of complex structures, on a closed oriented surface $M^{2}$. Of course, this has been widely studied, see e.g. [5]. In this case, the $L^{2}$ metric on $\mathscr{E}$ is known as the Weil-Petersson metric. One has the following basic trichotomy for the structure of $\mathscr{E}$ :

(i) $\lambda>0 \Rightarrow \mathscr{E}=\{\mathrm{pt}\}$, consisting of the unique constant curvature metric of volume 1 on $M=S^{2}$.

(ii) $\lambda=0 \Rightarrow \mathscr{E}=S L(2, \mathbf{Z}) \backslash S L(2, \mathbf{R}) / S O(2)$ is the space of flat metrics on the torus. The Weil-Petersson metric is the complete, bi-invariant metric of finite volume on $\mathscr{E}$.

(iii) $\lambda<0 \Rightarrow \mathscr{E}$ is the moduli space of hyperbolic metrics on a surface $\Sigma_{g}$ of genus $g>1$, and is the quotient of an open ball $B^{6 g-6}$ by the properly discontinuous action of the Teichmüller modular group $\Gamma_{g}$. $\mathscr{E}$

Received by the editors January 26, 1989 and, in revised form, May 1, 1989.

1980 Mathematics Subject Classification (1985 Revision). Primary 58G30, 58C25; Secondary 58E11, 53C55.

Partially supported by NSF Grant DMS 87-01137. 
is noncompact and incomplete, in fact of finite diameter, in the WeilPetersson metric, cf. [12]. The completion $\overline{\mathscr{E}}$ of $\mathscr{E}$ in the Weil-Petersson metric gives a compactification of the moduli space (the augmented moduli space [1]), with the ideal boundary points given by hyperbolic metrics with cusps, or Riemann surfaces with nodes, cf. [1,5]. This compactification agrees with the Mumford compactification in algebraic geometry.

The results described below show that many features of this trichotomy continue to hold on the space of Einstein metrics on a compact 4-manifold, at least if one allows mild singularities in the metrics.

DEFINITION. An Einstein orbifold $(V, g)$ is an orbifold with a finite number of singular points $\left\{p_{i}\right\} \in V$, each $p_{i}$ having a neighborhood $N_{i}$ homeomorphic to the cone $C\left(S^{3} / \Gamma\right)$ on a spherical space form $S^{3} / \Gamma, \Gamma \subset$ $S O(4)$. On the regular set $V_{0}=V-\left\{p_{i}\right\}, g$ defines a smooth Einstein metric, and when lifted to the universal cover $N_{i}-p_{i} \cong B^{4}-0, g$ is required to extend smoothly over $\{0\}$. We note that $V$ may be given the structure of a real-analytic variety. Typical examples are of course the (singular) flat metrics on the cones $C\left(S^{3} / \Gamma\right)$.

An orbifold-singular Einstein metric on a smooth 4-manifold $M$ is a smooth symmetric 2-tensor on $M$ of the form $\pi^{*}(g)$, where $\pi: M \rightarrow V$ is a surjective real analytic map to an Einstein orbifold $(V, g)$, such that if $M_{0}=\pi^{-1}\left(V_{0}\right)$, then $\left.\pi\right|_{M_{0}}$ is a diffeomorphism onto $V_{0}$ and $\pi^{-1}\left\{p_{i}\right\}$ is a connected, 2-dimensional, real-analytic subvariety $D_{i}$ of $M$ (possibly reducible), for each $i$; in particular, $N_{i}-p_{i} \approx N\left(D_{i}\right)-D_{i}$, where $N\left(D_{i}\right)$ is a neighborhood $D_{i}$ in $M$. Typical examples here are the maps giving nonsingular, complex resolutions of the singular spaces $C^{2} / \Gamma, \Gamma \subset S U(2)$, i.e. resolutions of rational double points.

In contrast to the 2-dimensional case, the space $\mathscr{E}$ is rarely complete, i.e. there exist sequences of Einstein metrics which are bounded in $L^{2}$, without convergent subsequences in $\mathscr{E}$, (even if $\lambda \geq 0$ ). However, the limits are well-behaved.

THEOREM A. Let $\mathscr{C}^{\lambda}, \lambda \in \mathbf{R}$, be a connected component of the moduli space of $\lambda$-Einstein metrics, $\operatorname{Ric}(g)=\lambda \cdot g$, on a compact, oriented 4-manifold $M$.

I. If $\lambda>0$, the closure $\overline{\mathscr{C}^{\lambda}}$ of $\mathscr{C}^{\lambda}$ in the $L^{2}$ metric is a compact Hausdorff space consisting of regular and orbifold singular $\lambda$-Einstein metrics on $M$. Locally, $\overline{\mathscr{C}^{\lambda}}$ is a quotient of a finite-dimensional real-analytic variety by a compact group action. The $L^{2}$ metric extends to a complete metric on the compactification $\overline{\mathscr{C} \lambda}$.

II. If $\lambda=0$, the closure $\overline{\mathscr{C}^{0}}$ of $\mathscr{C}^{0}$ in the $L^{2}$ metric is a complete, generally noncompact, Hausdorff space, with otherwise the same properties as I above. The function, diameter ${ }^{-1}: \overline{\mathscr{C}}^{0} \rightarrow \mathbf{R}$ is a proper exhaustion function on $\overline{\mathscr{C}^{0}}$.

III. If $\lambda<0$, the closure $\overline{\mathscr{C}^{\lambda}}$ of $\mathscr{C}^{\lambda}$ in the $L^{2}$ metric is a complete, Hausdorff space with frontier $\partial \mathscr{C}^{\lambda}$ consisting of two parts $\partial \mathscr{C}^{\lambda}=\partial_{0} \mathscr{C}^{\lambda} \cup \partial_{\infty} \mathscr{C}^{\lambda}$ given as follows:

(i) An element of $\partial_{0} \mathscr{C}^{\lambda}$ is an orbifold singular $\lambda$-Einstein metric on $M$, of volume 1 and bounded diameter. The partial completion $\mathscr{C}^{\lambda} \cup \partial_{0} \mathscr{C}^{\lambda}$ has the same local structure as I above and is locally complete in the $L^{2}$ metric. 
(ii) An element of $\partial_{\infty} \mathscr{C}^{\lambda}$ is a finite number of complete, noncompact (possibly orbifold singular), $\lambda$-Einstein 4-manifolds $N_{i}$, with $\operatorname{vol} N_{i} \leq 1$, diameter $N_{i}=\infty$. Further, $\Sigma$ vol $N_{i}=1$ and there are embeddings $N_{i} \subset M$ for each $i$, with disjoint images.

REMARKs. I. It is not true in general that $\mathscr{C}^{\lambda}$, for $\lambda>0$, is compact as in the 2-dimensional case. This was first shown by the examples of Tian-Yau [14], (these remain the only examples to date). In certain special cases, e.g. $M=S^{4}$ or $\mathbf{C P}^{2}$, it is known [2] that $\mathscr{C}^{\lambda}, \lambda>0$, is compact (in the $C^{\infty}$ topology).

II. Again, examples such as flat metrics on the torus $T^{4}$ show that $\overline{\mathscr{C}}$ is generally noncompact, (cf. also the discussion for K3 surfaces below). A divergent sequence $\left\{g_{i}\right\}$ (w.r.t. the $L^{2}$ metric) in $\overline{\mathscr{C}}{ }^{0}$ gives rise to a collapse of $M$ in the sense of [8], on the complement (metrically) of a finite number of points $\left\{p_{j}\right\} \in M$. More precisely, the sectional curvature $K_{i}$ of $g_{i}$ remains uniformly bounded on metrically compact subsets of $M-\left\{p_{j}\right\}$, while the injectivity radius converges to zero at every point of $M$ and $\operatorname{diam}_{M}\left(g_{i}\right) \rightarrow \infty$ as $i \rightarrow \infty$. This behavior is similar to the degeneration of flat metrics on the 2-torus. At the points $\left\{p_{j}\right\}$, the sequence develops orbifold singularities.

III. The manifolds $N_{i}$ in $\partial_{\infty} \mathscr{C}^{\lambda}$ are of course analogous to the cusps that are formed in the 2-dimensional case. If $\left\{g_{i}\right\}$ converges, in the $L^{2}$ metric, to a point on $\partial_{\infty} \mathscr{C}^{\lambda}$, then there are (finitely many) open domains $N_{k} \subset M$ such that $\left\{g_{i}\right\}$ converges, smoothly away from any orbifold singularities, to a complete, (orbifold-singular) $\lambda$-Einstein metric on $N_{k}$. The complement $M-\bigcup N_{k}$ is a set of measure zero, which collapses, in the sense of [8], under the sequence $\left\{g_{i}\right\}$.

Although the closure $\overline{\mathscr{C} \lambda}$ is complete in the $L^{2}$ metric, it is unknown in general if $\overline{\mathscr{C} \lambda}, \lambda<0$, is compact, as in dimension 2 . However, if $\overline{\mathscr{C} \lambda}$ is noncompact, then any divergent sequence in $\overline{\mathscr{C} \lambda}$ would have to collapse as in II above.

To a certain extent, these results are also valid for moduli spaces of Kähler-Einstein metrics on complex surfaces. These furnish the richest class of examples, via the solution of the Calabi conjecture [17] and, in the positive case, via [14]. The uniqueness of a Kähler-Einstein metric in a given Kähler class leads one to attempt to relate these results to the moduli spaces of Kähler classes, and thus also to the moduli space of complex structures on a complex surface with $c_{1}$ definite or zero. However, it is difficult in general to obtain control and convergence of the complex structures on $M$ from the Kähler-Einstein metrics.

The case of $\mathrm{K} 3$ surfaces has been worked out from this point of view and is particularly enlightening. Let $X^{4}$ be a smooth manifold diffeomorphic to a $\mathrm{K} 3$ surface. Thus, $X$ is simply connected and $L \equiv H^{2}(X, \mathbf{Z})$ is the free abelian integral lattice of rank 22 in $\mathbf{R}^{22} \cong H^{2}(X, \mathbf{R})$. The cup product gives a nondegenerate bilinear form on $H^{2}(X, \mathbf{R})$, of type $(3,19)$, and we let $T=\operatorname{Aut}(L)$ be the group of automorphisms of the lattice $L$, preserving the cup-form. Let $G_{3}^{+}=S O(3,19) / S O(3) \times S O(19)$ be the Grassmannian 
of oriented positive definite 3-planes in $H^{2}(X, \mathbf{R})$; then $T$ acts properly discontinuously (but not freely) on $G_{3}^{+}$and the quotient $\mathscr{G}=T \backslash G_{3}^{+}$is a locally symmetric orbifold, of finite volume in the canonical bi-invariant metric $g_{0}$ on $G_{3}^{+}$.

There is a natural period map [4],

$$
P: \mathscr{C}^{0}(X) \rightarrow \mathscr{G}
$$

where $P(g)$ is the class of the oriented positive definite 3-plane in $H^{2}(X, \mathbf{R})$ spanned by the three self-dual harmonic forms determined by $[g] \in \mathscr{C}^{0}(X)$. We show that $\overline{\mathscr{C}^{0}}(X)$ is a smooth orbifold and the period map $P$ extends smoothly over $\overline{\mathscr{C}^{0}}(X)$ to give a map $\bar{P}$ into $\mathscr{G}$. One obtains

THeroem B. The map $\bar{P}: \overline{\mathscr{C}^{0}}(X) \rightarrow \mathscr{G}$ is an isometry between $\overline{\mathscr{C}^{0}}(X)$, endowed with the $L^{2}$ metric, and the bi-invariant metric $g_{0}$ on the locally symmetric orbifold $\mathscr{G}$.

As a consequence, one obtains immediately and naturally the Surjectivity Theorem of [15] and a form of the Global Torelli Theorem of [7], for Kählerian K3 surfaces. The proof of these corollaries does not require, for instance, results on the density of the period map or the Torelli theorem for Kummer or algebraic K3 surfaces. The methods of proof are intrinsic from real differential geometry, except for the use of the Kodaira-Spencer theory of Kähler deformations and the solution of the Calabi conjecture.

We note that a proof of a (weaker) version of Theorem B is given in [10] (with some details omitted). The proof of [10] follows the classical proofs of the Torelli and Surjectivity theorems for K3 surfaces, and uses heavily the results of $[7,15]$ and especially [13].

Much of the initial work for these results appears in [2]; in particular, a large part of the proof of Theorem $A(I)$ appears there. The basic reason why these results are restricted to 4-manifolds is that the ChernGauss-Bonnet formula for the Euler characteristic is particularly strong on Einstein 4-manifolds, namely $\chi(M)=\left(1 / 8 \pi^{2}\right) \int|R|^{2}$, where $R$ is the full curvature tensor. The orbifold singularities that arise in the limit of a sequence of Einstein metrics are the loci where the curvature 'blows up', i.e. goes to infinity. As in [16], one shows this can happen only in a neighborhood of a finite number of points in $M$, by the use of elliptic differential inequalities satisfied by the curvature, and by control of the Sobolev constant of $M$. On the other hand, on domains where the curvature remains bounded, one has smooth convergence by the Gromov compactness theorem. The main ingredients necessary to produce the results above are then to obtain control on the structure of the singular set for the orbifold projections $\pi: M \rightarrow V$, and more importantly, to control the local Sobolev constants for domains in $M$ in terms of the $L^{2}$ metric on $\mathscr{E}$. This allows one to essentially localize the techniques used above. The details of these, as well as further results will appear in [3]. 


\section{REFERENCES}

1. W. Abikoff, The real analytic theory of Teichmüller space, Lecture Notes in Math., vol 820, Springer-Verlag, Berlin and New York, 1980.

2. M. Anderson, Ricci curvature bounds and Einstein metrics on compact manifolds, J. Amer. Math Soc. 2 (1989).

3. _,$L^{2}$ structure of moduli spaces of Einstein metrics on 4-manifolds (in preparation).

4. A. Beauville et al., Geometrie des surfaces K3: Modules et Periodes, Astérisque 126 (1985).

5. L. Bers, Finite dimensional Teichmüller spaces and generalizations, Bull. Amer. Math. Soc. (N.S.) 5 (1981), 131-172.

6. A. Besse, Einstein manifolds, Ergebnisse der Math. 3. Folge, Band 10, Springer-Verlag, Berlin and New York, 1987.

7. D. Burns and M. Rapaport, On the Torelli problem for Kählerian K3 surfaces, Ann. Sci. École Norm. Sup. 8 (1975), 235-274.

8. J. Cheeger and M. Gromov, Collapsing Riemannian manifolds while keeping their curvature bounded. I, J. Differential Geom. 23 (1986), 309-346.

9. M. Gromov, Structures metriques pour les variétiés Riemanniennes, Cedic/Nathan Fernand, (1981).

10. R. Kobayashi and A. Todorov, Polarized period map for generalized K3 surfaces and the moduli of Einstein metrics, Tôhoku Math. J. 39 (1987), 341-363.

11. N. Koiso, Einstein metrics and complex structures, Invent. Math. 73 (1983), 71-106.

12. H. Masur, The extension of the Weil-Petersson metric to the boundary of Teichmüller space, Duke Math. J. 43 (1975), 623-635.

13. D. Morrison, Some remarks on the moduli of $\mathrm{K} 3$ surfaces, Classification of Algebraic Manifolds, Progress in Math., 39, Birkhauser, (1983), 303-332.

14. G. Tian and S.-T. Yau, Kähler-Einstein metrics on complex surfaces with $C_{1}>0$, Comm. Math. Phys. 112 (1987), 175-203.

15. A. Todorov, Applications of the Kähler-Einstein-Calabi-Yau metric to moduli of $\mathrm{K} 3$ surfaces, Invent. Math. 61 (1980), 251-265.

16. K. Uhlenbeck, Removable singularities in Yang-Mills fields, Comm. Math. Phys. 83 (1982), 11-30.

17. S. -T. Yau, On the Ricci curvature of a compact Kähler manifold and the complex Monge-Ampere equation, Comm. Pure Appl. Math. 31 (1978), 339-441.

Department of Mathematics, SUNY at Stony Brook, Stony Brook, New York 11794-3651 
